\title{
Element for Beam Dynamic Analysis Based on Analytical Deflection Trial Function
}

\author{
Qiongqiong Cao, ${ }^{1}$ Min Ding, ${ }^{1}$ Xiugen Jiang, ${ }^{1}$ Jinsan Ju, ${ }^{1}$ Hongzhi Wang, ${ }^{1}$ and Peng Zhang ${ }^{1,2}$ \\ ${ }^{1}$ College of Water Resources \& Civil Engineering, China Agricultural University, Beijing 100083, China \\ ${ }^{2}$ China Aerospace Construction Group Co., Ltd., Beijing 100071, China \\ Correspondence should be addressed to Xiugen Jiang; jiangxg@cau.edu.cn
}

Received 18 September 2014; Accepted 15 December 2014

Academic Editor: Chenfeng Li

Copyright ( 2015 Qiongqiong Cao et al. This is an open access article distributed under the Creative Commons Attribution License, which permits unrestricted use, distribution, and reproduction in any medium, provided the original work is properly cited.

For beam dynamic finite element analysis, according to differential equation of motion of beam with distributed mass, general analytical solution of displacement equation for the beam vibration is obtained. By applying displacement element construction principle, the general solution of displacement equation is conversed to the mode expressed by beam end displacements. And taking the mode as displacement trial function, element stiffness matrix and element mass matrix for beam flexural vibration and axial vibration are established, respectively, by applying principle of minimum potential energy. After accurate integral, explicit form of element matrix is obtained. The comparison results show that the series of relative error between the solution of analytical trial function element and theoretical solution is about $1 \times 10^{-9}$ and the accuracy and efficiency are superior to that of interpolation trial function element. The reason is that interpolation trial function cannot accurately simulate the displacement mode of vibrating beam. The accuracy of dynamic stiffness matrix method is almost identical with that of analytical trial function. But the application of dynamic stiffness matrix method in engineering is limited. The beam dynamic element obtained in this paper is analytical and accurate and can be applied in practice.

\section{Introduction}

Dynamic structural analysis is essential in structure engineering design. It is especially important for large-scale structure in earthquake area, such as high-rise buildings, dam, hydropower station, oil pipelines, and gas pipelines. Dynamic problem of beam structure is common in engineering. Vibration happens at beam under earthquake, gasliquid flows, and impact. Resonance occurs when frequency of external load is close to natural frequency of structure. It is a great threat for structure safety. Therefore, perfecting accuracy and efficiency of dynamic structural analysis is to ensure structure safety and reliability.

A large number of theories and methods for dynamic structural analysis have been suggested. The analysis methods include direct solving method, energy method, and numerical method. Clough and Penzien [1] have obtained analytical solution of displacement equation for flexural vibrating beam and presented the first three-order vibration mode and the corresponding frequency of cantilever beam and simply supported beam. Jin [2] provided analytical solution of Timoshenko beam clamped two ends and subjected to uniformly distributed load according to different jump condition. Guo et al. [3] established structure element property matrix by energy principle considering flexural and torsional deformation of T-beam, and the effect of bridge local member (such as diaphragm plate). Fang and Wang [4] attained beam natural vibration frequency by analyzing dynamic property of external prestressing beam using energy method, and the solution has a better match with numerical solution. Lou and Hong [5] derived the approximation analysis technique for dynamic characteristics of the prestressed beam by applying the mode perturbation method. Carrer et al. [6] analyzed the dynamic behavior of Timoshenko beam by using boundary element method. Wu [7] analyzed the dynamic behavior of two-dimension frame with stiffening bar randomly distributed by using elasticrigid composite beam element. De Rosa et al. [8] studied 
the dynamic behavior of slender beam with concentrated mass at beam end, and numerical solution of frequency equation was obtained. Among them, numerical analysis represented by finite element is the main and efficient method for dynamic analysis (such as natural vibration analysis and forced vibration analysis). Finite element method was firstly proposed by Clough [9] in an article about plane elastic problem, and it is perfect in theory as a numerical method.

At present, the methods of constructing dynamic element for beam include dynamic stiffness matrix method, Galerkin method, Ritz method, energy variation method. Dynamic stiffness matrix can accurately solve differential equation of motion according to initial displacement field without any assumption, and then accurate results can be obtained irrespective of element number. This method was proposed by Koloušek [10]. To gain more accurate results, stiffness matrix of tensile torsion bar and Euler beam about frequency, that is, dynamic stiffness matrix, was firstly derived from analytical solution for studying vibration characteristics of plane truss. A lot of work on the research and development of dynamic stiffness matrix method was also done by Long and Bao [11], Hashemi and Richard [12], Chen et al. [13], Banerjee et al. [14], and Banerjee et al. [15]. Shavezipur and Hashemi [16] put forward an accurate finite element method. In this method, closed form solution of differential equation of beam not coupling flexural vibration torsional vibration was obtained by merging Galerkin weighted residual method and dynamic stiffness matrix (DSM). Result of dynamic stiffness matrix is more accurate, but analytical solution of differential equation cannot be derived when structure load or displacement boundary condition is too complex. Then dynamic stiffness matrix method is not suitable any more. According to principle of virtual displacement, a large number of research achievements on dynamic analysis of thin-walled open section beam, elastic foundation beam, and composite beam have been conducted by Chopra [17], $\mathrm{Hu}$ and Dai [18], Wang et al. [19], Hashemi and Richard [20], and Pagani et al. [21]. Nabi and Ganesan [22] put forward a finite element method based on free vibration analysis theory of composite beam with the first order shear deformation. Zhao and Chen [23] developed the dynamic analysis of a unified stochastic variational principle and the corresponding stochastic finite element method via the instantaneous minimum potential energy principle and the small parameter perturbation technique. On the basis of energy variation principle, Wang et al. [24] derived governing differential equation and natural boundary condition of dynamic response for I-shaped beam and obtained closed solution of the corresponding generalized dynamic displacement.

Accuracy and efficiency of beam element depend on beam displacement trial function by applying potential energy variation principle to constructing beam displacement element. For current beam element, cubic polynomial displacement mode is used to obtain a series of static and dynamic element widely applied to the software, such as ANSYS and NASTRAN. For dynamic analysis, vibrating beam displacement mode has a big difference from polynomial mode. Precision requirements cannot be met by taking polynomial function as vibrating beam displacement trail function. The basic analytical solution is used as the element trial function in analytical trial function method. Discrete finite element method takes advantage of analytical solution. It embodies the superiority of trial function using basic analytical solution.

This paper focuses on constructing element for beam dynamic analysis using analytical deflection trail function based on variational method of principle of minimum potential energy and displacement element construction theory. The fruits are useful to beam dynamic analysis.

\section{Displacement Trial Function for Beam Element}

Selecting displacement trial function is one of the main contents of constructing displacement element. Appropriate displacement trial function should be in accordance with element deformation behavior and should be easy for integral of element energy functional. Element accuracy is determined by the accuracy of displacement trial function. The corresponding functional integral has a direct effect on element calculation efficiency and accuracy.

With regard to beam element, displacement mode based on interpolation function is used for element displacement trial function of all kinds of problems. Linear polynomial Lagrange interpolation function meeting the continuity condition at $C_{0}$ is used for axial displacement. Cubic polynomial Hermit interpolation function meeting the continuity condition at $C_{1}$ is used for flexural deformation.

Take flexural deformation, for example; for the static problems of uniform cross section beam subjected to uniform distributed load, $E I\left(d^{4} w(x) / d x^{4}\right)=q$ is stiffness equilibrium equation about deflection $w(x)$. Here, $E I$ is beam section flexural stiffness and $q$ is the uniform distributed load. The accurate solution of this equation is a cubic polynomial. Cubic polynomial Hermit interpolation function meeting the continuity condition of beam end displacement is actual displacement of element. And the corresponding potential energy functional has analytic, derivable, integrable solution. For this reason, static beam element derived from Hermit interpolation shape function is accurate element.

However, for dynamic and nonlinear straight beam and every nonlinear beam, deflection equation is not cubic polynomial due to the change of equilibrium differential equation mode. Therefore, element constructed by Hermit interpolation trial function is an approximate element. The key of constructing accurate element for all kinds of problems of beam is to search analytical trial function having functional integrability.

\section{Analytical Trial Function of Displacement for Vibrating Beam Element}

The method to construct analytical trial function of displacement for vibrating beam element is as follows:

(1) to deduce the general solution of displacement equation for beam vibration containing undetermined 


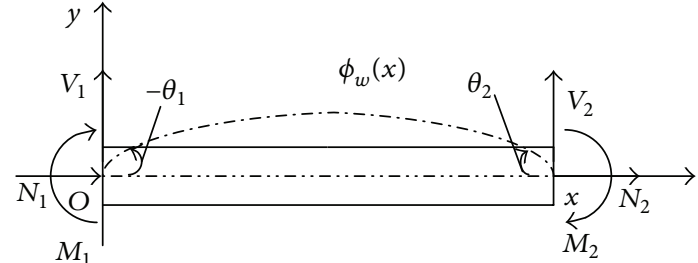

FIGURE 1: Coordinate system and parameter positive direction.

parameters according to differential equation of equilibrium for beam vibration;

(2) to determine the undetermined parameters in displacement equation according to displacement condition of vibrating beam end;

(3) to write out displacement equation for beam vibration expressed by beam end displacement, and then to obtain displacement trial function for vibrating beam.

To construct beam dynamic element, local coordinate as shown in Figure 1 is established. It is in accordance with general beam element. The positive direction of linear displacement of beam end and force is in accordance with coordinate direction, and the positive direction of rotation angle and moment is in accordance with clockwise direction.

\subsection{Governing Equation for Beam Free Vibration \\ and Displacement General Solution}

3.1.1. Beam Flexural Vibration Equation. Dynamic equilibrium governing equation for beam flexural vibration is expressed as [1]

$$
E I \frac{\partial^{4} v(x, t)}{\partial x^{4}}+\bar{m} \frac{\partial^{2} v(x, t)}{\partial t^{2}}=0 .
$$

In (1), $v(x, t)$ is the displacement response for beam flexural vibration.

By applying the method of separation of variables, analytical solution of deflection equation for beam flexural vibration is obtained [1]:

$$
\phi_{w}(x)=C_{1} \sin \alpha x+C_{2} \cos \alpha x+C_{3} \operatorname{sh} \alpha x+C_{4} \operatorname{ch} \alpha x .
$$

In (2), flexural vibration parameter $\alpha$ is connected with circular frequency for beam flexural vibration $\omega_{w}, \omega_{w}=$ $\alpha^{2} \sqrt{E I / \bar{m}}, E I$ is beam section flexural stiffness, and $\bar{m}$ is beam mass per unit length.

3.1.2. Beam Axial Vibration Equation. Dynamic equilibrium governing equation for beam axial vibration is expressed as [1]

$$
E A \frac{d^{2} u}{d x^{2}}-\bar{m} \frac{\partial^{2} u}{\partial t^{2}}=0
$$

In (3), $u(x, t)$ is the displacement response for beam axial vibration.
By applying the method of separation of variables, analytical solution of deflection equation for beam axial vibration is obtained [1]:

$$
\phi_{u}(x)=D_{1} \cos \beta x+D_{2} \sin \beta x .
$$

In (4), axial vibration parameter $\beta$ is connected with circular frequency for beam axial vibration $\omega_{u}, \omega_{u}=\beta \sqrt{E A / \bar{m}}$, and $E A$ is beam section tensile (compressive) rigidity.

\subsection{Analytical Trial Function of Displacement for Dynamic Beam Element}

\subsubsection{Analytical Trial Function for Beam Flexural Vibration}

(1) Parameter Determination of Displacement Function. Equation (2) can be further expressed as

$$
\phi_{w}(x)=\sum_{i=1}^{4} \varphi_{i}(x) C_{i}=\{\varphi\}\{C\},
$$

where

$$
\begin{gathered}
\{C\}=\left\{\begin{array}{llll}
C_{1} & C_{2} & C_{3} & C_{4}
\end{array}\right\}^{T}, \\
\{\varphi\}=\left\{\begin{array}{lllll}
\varphi_{1}(x) & \varphi_{2}(x) & \varphi_{3}(x) & \varphi_{4}(x)
\end{array}\right\} \\
=\left\{\begin{array}{llll}
\sin \alpha x & \cos \alpha x & \operatorname{sh} \alpha x & \operatorname{ch} \alpha x
\end{array} .\right.
\end{gathered}
$$

Beam end displacement is defined as $\left\{\delta_{w}\right\}^{e}=$ $\left\{\begin{array}{llll}\omega_{1} & \theta_{1} & \omega_{2} & \theta_{2}\end{array}\right\}^{T}$.

According to (5), beam end displacement becomes

$$
\begin{aligned}
\left\{\delta_{w}\right\}^{e}= & {\left[\begin{array}{cccc}
\varphi_{1}(0) & \varphi_{2}(0) & \varphi_{3}(0) & \varphi_{4}(0) \\
-\varphi_{1}^{\prime}(0) & -\varphi_{2}^{\prime}(0) & -\varphi_{3}^{\prime}(0) & -\varphi_{4}^{\prime}(0) \\
\varphi_{1}(l) & \varphi_{2}(l) & \varphi_{3}(l) & \varphi_{4}(l) \\
-\varphi_{1}^{\prime}(l) & -\varphi_{2}^{\prime}(l) & -\varphi_{3}^{\prime}(l) & -\varphi_{4}^{\prime}(l)
\end{array}\right] } \\
& \cdot\left\{\begin{array}{l}
C_{1} \\
C_{2} \\
C_{3} \\
C_{4}
\end{array}\right\}=[A]\{C\} .
\end{aligned}
$$

If $\lambda=\alpha l$, with (6), [A] can be expressed as

$$
[A]=\left[\begin{array}{cccc}
0 & 1 & 0 & 1 \\
-\alpha & 0 & -\alpha & 0 \\
\sin \lambda & \cos \lambda & \operatorname{sh} \lambda & \operatorname{ch} \lambda \\
-\alpha \cos \lambda & \alpha \sin \lambda & -\alpha \operatorname{ch} \lambda & -\alpha \operatorname{sh} \lambda
\end{array}\right]
$$

With (7), undetermined parameter of displacement function is expressed as

$$
\{C\}=[A]^{-1}\left\{\delta_{w}\right\}^{e} .
$$


To perform matrix inversion, then

$$
[A]^{-1}=\left[\begin{array}{llll}
c_{11} & c_{12} & c_{13} & c_{14} \\
c_{21} & c_{22} & c_{23} & c_{24} \\
c_{31} & c_{32} & c_{33} & c_{34} \\
c_{41} & c_{42} & c_{43} & c_{44}
\end{array}\right]
$$

where

$$
\begin{gathered}
c_{11}=-c_{31}=\frac{\cos \lambda \sinh \lambda+\cosh \lambda \sin \lambda}{S}, \\
c_{12}=-\frac{\cos \lambda \cosh \lambda+\sinh \lambda \sin \lambda-1}{\alpha S}, \\
c_{13}=-c_{33}=-\frac{\sin \lambda+\sinh \lambda}{S}, \\
c_{14}=-c_{34}=-\frac{\cosh \lambda-\cos \lambda}{\alpha S}, \\
c_{21}=\frac{1-\sinh \lambda \sin \lambda+\cos \lambda \cosh \lambda}{S}, \\
c_{22}=-c_{42}=\frac{\cosh \lambda \sin \lambda-\cos \lambda \sinh \lambda}{S}, \\
c_{23}=-c_{43}=\frac{\cosh \lambda-\cos \lambda}{S}, \\
c_{41}=\frac{\cos \lambda \cosh \lambda+\sin \lambda \sinh \lambda-1}{S}, \\
c_{24}=-c_{44}=\frac{\sinh \lambda-\sin \lambda}{\alpha S}, \\
S=2(\cos \lambda \cosh \lambda-1) . \sinh \lambda \sin \lambda-\cos \lambda \cosh \lambda \\
\alpha S
\end{gathered},
$$

(2) Element Displacement Shape Function. By substituting (9) into (5), displacement function for element flexural vibration expressed by beam end displacement can be obtained:

$$
\phi_{w}(x)=\{\varphi\}[A]^{-1}\left\{\delta_{w}\right\}^{e}
$$

If

$$
\left[N_{w}\right]=\{\varphi\}[A]^{-1}
$$

(12) becomes

$$
\phi_{w}(x)=\left[N_{w}\right]\left\{\delta_{w}\right\}^{e}
$$

$\left[N_{w}\right]$ is flexural vibration displacement trial function for dynamic beam element. With (13), it can be expressed as

$$
\left[N_{w}\right]=\{\varphi\}[A]^{-1}=\left[\begin{array}{llll}
N_{1} & N_{2} & N_{3} & N_{4}
\end{array}\right]
$$

where $N_{1}, N_{2}, N_{3}$, and $N_{4}$ are deflection shape functions. After matrix operation, they can be expressed as

$$
\begin{aligned}
N_{1}=\frac{1}{S}[ & \sinh \lambda \sin (\alpha x-\lambda)+\cosh \lambda \cos (\alpha x-\lambda) \\
& -\cos (\alpha x)+\cos \lambda \cosh (\alpha x-\lambda) \\
& -\sin \lambda \sinh (\alpha x-\lambda)-\cosh (\alpha x)],
\end{aligned}
$$

$$
\begin{aligned}
N_{2}=\frac{1}{\alpha S}[ & -\cosh \lambda \sin (\alpha x-\lambda)-\sinh \lambda \cos (\alpha x-\lambda) \\
& +\sin (\alpha x)+\sinh (\alpha x)-\sin \lambda \cosh (\alpha x-\lambda) \\
& -\cos \lambda \sinh (\alpha x-\lambda)],
\end{aligned}
$$$$
N_{3}=\frac{1}{S}[-\sin (\alpha x) \sinh \lambda-\cos (\alpha x-\lambda)
$$$$
+\cos (\alpha x) \cosh \lambda-\cosh (\alpha x-\lambda)
$$$$
+\sin \lambda \sinh (\alpha x)+\cosh (\alpha x) \cos \lambda],
$$$$
N_{4}=\frac{1}{\alpha S}[-\sin (\alpha x) \cosh \lambda+\sin (\alpha x-\lambda)
$$$$
+\cos (\alpha x) \sinh \lambda+\sinh (\alpha x-\lambda)
$$$$
-\sinh (\alpha x) \cos \lambda+\cosh (\alpha x) \sin \lambda] \text {. }
$$

\subsubsection{Analytical Trial Function for Beam Axial Vibration}

(1) Parameter Determination of Displacement Function. Equation (4) can be further expressed as

$$
\phi_{u}(x)=\sum_{i=1}^{4} \psi_{i}(x) D_{i}=\{\psi\}\{D\},
$$

where

$$
\begin{gathered}
\{D\}=\left\{\begin{array}{ll}
D_{1} & D_{2}
\end{array}\right\}^{T}, \\
\{\psi\}=\left\{\begin{array}{ll}
\psi_{1}(x) & \psi_{2}(x)
\end{array}\right\} \\
=\left\{\begin{array}{ll}
\cos \beta x & \sin \beta x
\end{array}\right\} .
\end{gathered}
$$

Beam end axial displacement is defined as $\left\{\delta_{u}\right\}^{e}=$ $\left\{\begin{array}{ll}u_{1} & u_{2}\end{array}\right\}^{T}$.

According to (17), beam end axial displacement is obtained:

$$
\left\{\delta_{u}\right\}^{e}=\left[\begin{array}{ll}
\psi_{1}(0) & \psi_{2}(0) \\
\psi_{1}(l) & \psi_{2}(l)
\end{array}\right]\left\{\begin{array}{l}
D_{1} \\
D_{2}
\end{array}\right\}=[B]\{D\} .
$$

If $\gamma=\beta l$, with (18), [B] can be expressed as

$$
[B]=\left[\begin{array}{cc}
1 & 0 \\
\cos \gamma & \sin \gamma
\end{array}\right] \text {. }
$$

With (19), undetermined parameter of axial displacement function is expressed as

$$
\{D\}=[B]^{-1}\left\{\delta_{u}\right\}^{e} .
$$


To perform matrix inversion, then

$$
[B]^{-1}=\left[\begin{array}{cc}
1 & 0 \\
-\frac{\cos \gamma}{\sin \gamma} & \frac{1}{\sin \gamma}
\end{array}\right] \text {. }
$$

(2) Element Displacement Shape Function. By substituting (21) into (17), the following can be obtained:

$$
\phi_{u}(x)=\{\psi\}[B]^{-1}\left\{\delta_{u}\right\}^{e} .
$$

If

$$
\left[N_{u}\right]=\{\psi\}[B]^{-1}
$$

(23) becomes

$$
\phi_{u}(x)=\left[N_{u}\right]\left\{\delta_{u}\right\}^{e}
$$

$\left[N_{u}\right]$ is axial vibration displacement trial function for dynamic beam element. With (23), it can be expressed as

$$
\left[N_{u}\right]=\{\psi\}[B]^{-1}=\left[\begin{array}{ll}
N_{5} & N_{6}
\end{array}\right]
$$

where $N_{5}, N_{6}$ are axial vibration displacement shape functions. After matrix operation, they can be expressed as

$$
\begin{aligned}
& N_{5}=\frac{\cos \beta x-\sin \beta x \cos \gamma}{\sin \gamma}, \\
& N_{6}=\frac{\sin \beta x}{\sin \gamma} .
\end{aligned}
$$

\section{Element Potential Energy Functional and Variation}

Finite element formulation of dynamic beam element is constructed by principle of minimum potential energy and analytical trial function.

4.1. Potential Energy Functional. In the light of potential energy, functional of total potential energy is given by

$$
\begin{aligned}
\prod_{p}= & U+V \\
= & \frac{1}{2} \int_{0}^{l} E A \phi_{u}^{\prime 2}(x) d x+\frac{1}{2} \int_{0}^{l} E I \phi_{w}^{\prime \prime 2}(x) d x \\
& -\frac{1}{2} \int_{0}^{l} \bar{m} \omega_{u}^{2} \phi_{u}^{2}(x) d x-\frac{1}{2} \int_{0}^{l} \bar{m} \omega_{w}^{2} \phi_{w}^{2}(x) d x,
\end{aligned}
$$

where $U$ is element strain energy and $V$ is potential energy of inertia force.
With element displacement, the following can be obtained:

$$
\begin{aligned}
\prod_{p}= & \frac{1}{2} \int_{0}^{l} E A\left\{\delta_{u}\right\}^{e T}\left[N_{u}^{\prime}\right]^{T}\left[N_{u}^{\prime}\right]\left\{\delta_{u}\right\}^{e} d x \\
& +\frac{1}{2} \int_{0}^{l} E I\left\{\delta_{w}\right\}^{e T}\left[N_{w}^{\prime \prime}\right]^{T}\left[N_{w}^{\prime \prime}\right]\left\{\delta_{w}\right\}^{e} d x \\
& -\frac{1}{2} \int_{0}^{l} \bar{m} \omega_{u}^{2}\left\{\delta_{u}\right\}^{e T}\left[N_{u}\right]^{T}\left[N_{u}\right]\left\{\delta_{u}\right\}^{e} d x \\
& -\frac{1}{2} \int_{0}^{l} \bar{m} \omega_{w}^{2}\left\{\delta_{w}\right\}^{e T}\left[N_{w}\right]^{T}\left[N_{w}\right]\left\{\delta_{w}\right\}^{e} d x .
\end{aligned}
$$

4.2. Functional Variation and Transformation. In the light of principle of minimum potential energy, that is, $\partial \prod_{p} / \partial\{\delta\}^{e}=$ 0 , element equilibrium equation is obtained:

$$
[K]^{e}\{\delta\}^{e}-\omega^{2}[M]^{e}\{\delta\}^{e}=0,
$$

where $[K]^{e}$ and $[M]^{e}$ are dynamic stiffness matrix and mass matrix of beam element, respectively:

$$
\begin{aligned}
& {[K]^{e}=\int_{0}^{l} E A\left[N_{u}^{\prime}\right]^{T}\left[N_{u}^{\prime}\right] d x+\int_{0}^{l} E I\left[N_{w}^{\prime \prime}\right]^{T}\left[N_{w}^{\prime \prime}\right] d x} \\
& {\left[M_{w}\right]^{e}=\int_{0}^{l} \bar{m}\left[N_{u}\right]^{T}\left[N_{u}\right] d x+\int_{0}^{l} \bar{m}\left[N_{w}\right]^{T}\left[N_{w}\right] d x .}
\end{aligned}
$$

\section{Element Matrix}

Flexural vibration and axial vibration are independent of one another irrespective of large deformation. Element matrixes at flexural vibration and axial vibration are obtained by applying variation on flexural vibration displacement and axial vibration displacement, respectively.

\subsection{Element Matrix for Beam Flexural Vibration}

5.1.1. Stiffness Matrix. After matrix operation, element dynamic stiffness matrix for beam flexural vibration is expressed as

$$
\left[K_{w}\right]^{e}=H\left[\begin{array}{llll}
k_{w 11} & k_{w 12} & k_{w 13} & k_{w 14} \\
k_{w 21} & k_{w 22} & k_{w 23} & k_{w 24} \\
k_{w 31} & k_{w 32} & k_{w 33} & k_{w 34} \\
k_{w 41} & k_{w 42} & k_{w 43} & k_{w 44}
\end{array}\right],
$$

where

$$
\begin{gathered}
k_{w i j}=\frac{1}{H} \int_{0}^{l} N_{w i}^{\prime \prime} N_{w i}^{\prime \prime} d x \\
H=\frac{E I \alpha^{3} e^{-2 \lambda}}{G}, \\
G=16\left(1+\cos ^{2} \lambda \operatorname{ch}^{2} \lambda-2 \cos \lambda \operatorname{ch} \lambda\right) .
\end{gathered}
$$


After integral, each stiffness matrix coefficient can be obtained, such as

$$
\begin{aligned}
k_{w 11}= & (\cosh \lambda \sin \lambda+\cos \lambda \sin \lambda-\sinh \lambda \cosh \lambda \\
& \left.-2 \cosh ^{2} \lambda \sin \lambda \cos \lambda\right) \\
& \cdot\left(2 e^{2 \lambda}+e^{4 \lambda}+1\right) \\
& +4\left(e^{\lambda}-e^{3 \lambda}\right) \cosh \lambda(\sinh \lambda \sin \lambda+\cosh \lambda \cos \lambda) \\
& -2\left(1+e^{4 \lambda}\right) \cos \lambda \sin \lambda \cosh \lambda \sinh \lambda \\
& +4 e^{2 \lambda} \cos ^{2} \lambda(\lambda-\sinh \lambda \cosh \lambda) \\
& +\left(6 e^{2 \lambda}+e^{4 \lambda}+1\right) \cos \lambda \sinh \lambda \\
& +\left(1-e^{4 \lambda}-8 e^{2 \lambda} \lambda\right) \cos \lambda \cosh ^{4 \lambda}+4\left(e^{\lambda}+e^{3 \lambda}\right) \\
& +\left(\cos \lambda \sinh ^{3 \lambda} \cosh \lambda-\cos ^{2} \lambda \sinh \lambda-\sin \lambda\right. \\
& \left.+\cosh ^{2} \lambda \sin \lambda-\cos ^{2} \lambda \cosh ^{2} \lambda\right) \\
& +\left(4 \lambda e^{2 \lambda}+e^{4 \lambda}-1\right) \cosh ^{2} \lambda+\left(1-e^{4 \lambda}\right) \sin \lambda \sinh \lambda
\end{aligned}
$$

5.1.2. Mass Matrix. Mass matrix of element for beam flexural vibration is given by

$$
\left[M_{w}\right]=P\left[\begin{array}{llll}
m_{w 11} & m_{w 12} & m_{w 13} & m_{w 14} \\
m_{w 21} & m_{w 22} & m_{w 23} & m_{w 24} \\
m_{w 31} & m_{w 32} & m_{w 33} & m_{w 34} \\
m_{w 41} & m_{w 42} & m_{w 43} & m_{w 44}
\end{array}\right]
$$

where

$$
\begin{gathered}
m_{w i j}=\frac{1}{P} \int_{0}^{l} N_{w i} N_{w j} d x, \quad P=\frac{\bar{m} e^{-2 \lambda}}{G}, \\
G=16\left(1+\cos ^{2} \lambda \operatorname{ch}^{2} \lambda-2 \cos \lambda \operatorname{ch} \lambda\right) .
\end{gathered}
$$

After integral, each mass matrix coefficient can be obtained, such as

$$
\begin{aligned}
m_{w 11}=\alpha^{-1}[ & 4\left(e^{\lambda}+e^{3 \lambda}\right) \\
& \cdot\left(\cos ^{2} \lambda \sinh \lambda-\cos \lambda \sinh \lambda \cosh \lambda\right. \\
& \left.\quad-\sin \lambda \cosh ^{2} \lambda+\sin \lambda\right) \\
& +\left(4 \lambda e^{2 \lambda}+e^{4 \lambda}-1\right) \cosh ^{2} \lambda \\
& +\left(1-e^{4 \lambda}\right) \sin \lambda \\
& \cdot(\sinh \lambda-2 \cos \lambda \sinh \lambda \cosh \lambda)
\end{aligned}
$$

$$
\begin{aligned}
& +\left(e^{4 \lambda}-10 e^{2 \lambda}+1\right) \cos \lambda \sinh \lambda \\
& -\left(8 \lambda e^{2 \lambda}+e^{4 \lambda}-1\right) \cos \lambda \cosh \lambda \\
& +4\left(e^{3 \lambda}-e^{\lambda}\right) \\
& \cdot \cosh \lambda\left(\sin \lambda \sinh \lambda-\cos ^{2} \lambda+4 \cos \lambda \cosh \lambda\right) \\
& +\left(2 e^{2 \lambda}+e^{\lambda}+1\right)(\sin \lambda \cos \lambda-\sinh \lambda \cosh \lambda) \\
& +3 e^{2 \lambda} \cos ^{2} \lambda(4 \sinh \lambda \cosh \lambda+\lambda) \\
& +2\left(6 e^{2 \lambda}-1-e^{4 \lambda}\right) \sin \lambda \cos \lambda \cosh ^{2} \lambda \\
& \left.+\left(e^{4 \lambda}-14 e^{2 \lambda}+1\right) \sin \lambda \cosh \lambda\right] .
\end{aligned}
$$

5.2. Element Matrix for Beam Axial Vibration. After matrix operation, element dynamic stiffness matrix for beam axial vibration is expressed as

$$
\left[K_{u}\right]^{e}=E A c\left[\begin{array}{cc}
-\frac{\cos \gamma \sin \gamma+\gamma}{2\left(\cos ^{2} \gamma-1\right)} & \frac{\gamma \cos \gamma+\sin \gamma}{2\left(\cos ^{2} \gamma-1\right)} \\
\frac{\gamma \cos \gamma+\sin \gamma}{2\left(\cos ^{2} \gamma-1\right)} & -\frac{\cos \gamma \sin \gamma+\gamma}{2\left(\cos ^{2} \gamma-1\right)}
\end{array}\right] .
$$
by

Mass matrix of element for beam axial vibration is given

5.3. Dynamic Beam Element Matrix. Because of the independence of axial vibration and flexural vibration, 6 by 6 matrix is obtained by adding two rows and two columns to the above 4 by 4 matrix considering axial vibration without coupling of axial vibration and flexural vibration. It is element stiffness matrix.

Element stiffness matrix is given by

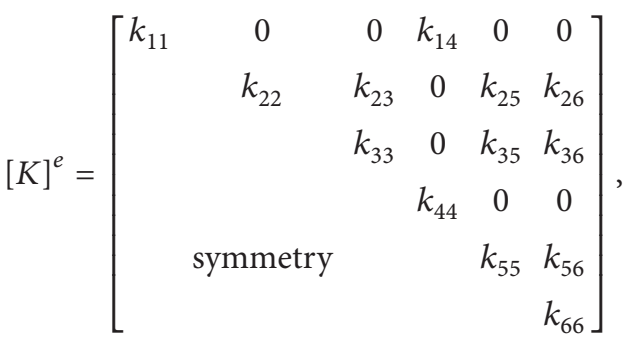


where

$$
\begin{aligned}
k_{11}=-\frac{E A c(\cos \gamma \sin \gamma+\gamma)}{2\left(\cos ^{2} \gamma-1\right)}, \\
k_{14}=\frac{E A c(\gamma \cos \gamma+\sin \gamma)}{2\left(\cos ^{2} \gamma-1\right)}, \\
k_{22}=H k_{w 11}, \\
k_{23}=H k_{w 12}, \\
k_{25}=H k_{w 13}, \\
k_{26}=H k_{w 14}, \\
k_{33}=H k_{w 22}, \\
k_{35}=H k_{w 23}, \\
k_{36}=H k_{w 24}, \\
k_{44}=-\frac{E A c(\cos \gamma \sin \gamma+\gamma)}{2\left(\cos ^{2} \gamma-1\right)} \\
k_{55}=H k_{w 33}, \\
k_{56}=H k_{w 34}, \\
k_{66}=H k_{w 44} .
\end{aligned}
$$

Element mass matrix is given by

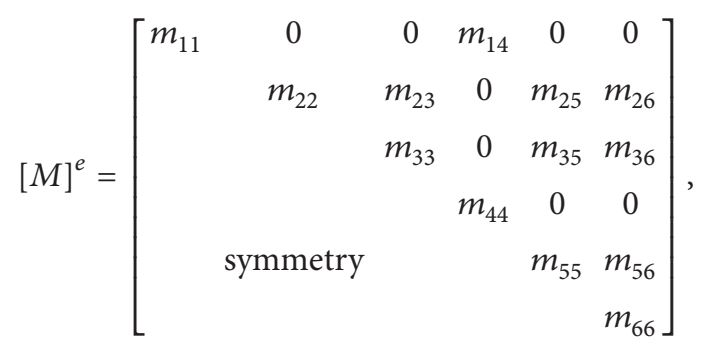

where

$$
\begin{gathered}
m_{11}=\frac{\bar{m}(\sin \gamma \cos \gamma-\gamma)}{2 c\left(\cos ^{2} \gamma-1\right)}, \\
m_{14}=\frac{\bar{m}(\gamma \cos \gamma-\sin \gamma)}{2 c\left(\cos ^{2} \gamma-1\right)}, \\
m_{22}=P m_{w 11}, \\
m_{23}=P m_{w 12} \\
m_{25}=P m_{w 13} \\
m_{26}=P m_{w 14} \\
m_{33}=P m_{w 22} \\
m_{35}=P m_{w 23} \\
m_{36}=P m_{w 24}
\end{gathered}
$$

$$
\begin{gathered}
m_{44}=\frac{\bar{m}(\sin \gamma \cos \gamma-\gamma)}{2 c\left(\cos ^{2} \gamma-1\right)}, \\
m_{55}=P m_{w 33}, \\
m_{56}=P m_{w 34}, \\
m_{66}=P m_{w 44} .
\end{gathered}
$$

\section{Example and Comparison}

To verify the dynamic beam element constructed by analytical trial function method, the comparisons between the calculation results of this element, general beam element, and theoretical solution are conducted.

\subsection{Calculation Model}

(1) Four Kinds of Typical Beams. Free vibration of four kinds of typical beams are analyzed, including cantilever beam, simply supported beam, one end clamped and another simply supported beam, and clamped-clamped beam.

(2) Structure and Material Parameters. The parameters of calculation model are as follows: beam dimension is $6 \mathrm{~m}$ $\times 0.2 \mathrm{~m} \times 0.3 \mathrm{~m}$, material elastic modulus $E=210 \mathrm{GPa}$, Poisson's ratio $v=0.3$, and material density $\rho=7800 \mathrm{~kg} / \mathrm{m}^{3}$.

(3) Theoretical Solution. According to theoretical equation of free vibration for distributed mass beam, base frequencies of free vibration for all kinds of beam are obtained by using analytic method [1].

(4) Dynamic Stiffness Matrix Method. According to governing equation of free vibration for distributed mass beam, stiffness coefficient [11] of beam vibration is provided, and then base frequencies of free vibration for all kinds of beam are obtained.

(5) Finite Element Model of General Beam Element. By using the software ANSYS, finite element model of beam vibration is established and modal analysis is conducted. Element BEAM3 is used to simulate beam. BEAM3 is general two-dimension elastic beam element and uniaxial element bearing tension, pressure, and bend. Every node has three degrees of freedom, that is, $x$-axis linear displacement, $y$-axis linear displacement, and $z$-axis angular displacement. Cubic polynomial interpolating function is used as its displacement trial function. Beam is divided into one beam element. Block Lanczos method is used for modal extraction.

(6) Model in This Paper. Equilibrium equation of beam flexural free vibration is given by

$$
K(\omega) \Delta(t)+M(\omega) \ddot{\Delta}(t)=0,
$$

where $K(\omega)$ is structure stiffness matrix with boundary displacement restraint and $M(\omega)$ is structure mass matrix with boundary displacement restraint. According to natural 
TABLE 1: Base frequency of beam free vibration and its comparison ( $\mathrm{rad} / \mathrm{s})$.

\begin{tabular}{|c|c|c|c|c|c|c|c|}
\hline \multirow{2}{*}{ Beam type } & \multirow{2}{*}{$\begin{array}{l}\text { Theoretical } \\
\text { solution }\end{array}$} & \multicolumn{2}{|c|}{$\begin{array}{l}\text { Dynamic stiffness matrix } \\
\text { method }\end{array}$} & \multicolumn{2}{|c|}{$\begin{array}{l}\text { Interpolation trial } \\
\text { function element }\end{array}$} & \multicolumn{2}{|c|}{$\begin{array}{c}\text { Analytical trial function } \\
\text { element }\end{array}$} \\
\hline & & $\begin{array}{l}\text { Absolute } \\
\text { value }\end{array}$ & $\begin{array}{c}\text { Relative error } \\
(\%)\end{array}$ & $\begin{array}{l}\text { Absolute } \\
\text { value }\end{array}$ & $\begin{array}{c}\text { Relative error } \\
(\%)\end{array}$ & $\begin{array}{l}\text { Absolute } \\
\text { value }\end{array}$ & $\begin{array}{c}\text { Relative error } \\
(\%)\end{array}$ \\
\hline Cantilever beam & 43.8875389 & 43.8875390 & $2.89 E-07$ & 44.0740 & 0.423 & 43.8875391 & $3.12 E-07$ \\
\hline $\begin{array}{l}\text { Simply supported } \\
\text { beam }\end{array}$ & 123.1941891 & 123.1941888 & $-8.59 E-08$ & 136.5902 & 10.874 & 123.1941889 & $-6.37 E-08$ \\
\hline $\begin{array}{l}\text { One end clamped and } \\
\text { another simply } \\
\text { supported beam }\end{array}$ & 192.4528355 & 192.4528348 & $-1.50 E-07$ & 255.4366 & 32.727 & 192.4528349 & $-1.53 E-07$ \\
\hline $\begin{array}{l}\text { Clamped-clamped } \\
\text { beam }\end{array}$ & 279.2673996 & 279.2673991 & $-1.32 E-07$ & 283.4156 & 1.485 & 279.2673992 & $-1.27 E-07$ \\
\hline
\end{tabular}

Note: calculation of eigenvalue and eigenvector cannot be conducted due to all DOFs of two ends of clamped-clamped beam being restrained. If the beam is divided into one element, semistructure is used for vibration simulation of clamped-clamped beam.

TABle 2: Comparison of different element results (relative error, \%).

\begin{tabular}{lcccc}
\hline Beam type & $\begin{array}{c}\text { Element number for analytical } \\
\text { trial function }\end{array}$ & \multicolumn{2}{c}{ Element number for interpolation trial function } \\
& 1 & 1 & 2 & 10 \\
\hline Cantilever beam & $3.12 E-07$ & 0.4249 & -0.0003 & -0.0489 \\
Simply supported beam & $-6.37 E-08$ & 10.8739 & 0.2909 & -0.0489 \\
One end clamped and another & $-1.53 E-07$ & 32.7269 & 0.8037 & -0.1018 \\
simply supported beam & $-1.27 E-07$ & 1.4854 & 1.4854 & -0.1169 \\
Clamped-clamped beam & & -0.1202 & -0.1278 \\
\hline
\end{tabular}

vibration governing equation of beam, beam natural vibration is harmonic vibration. The following assumption can be obtained:

$$
\Delta(t)=Y \sin \omega t
$$

Substituting (45) into (44) yields

$$
M^{-1}(\omega) K(\omega) Y=\omega^{2} Y
$$

Assuming $\eta=\omega^{2}$, then $E(\eta)=M^{-1}(\eta) K(\eta)$.

The following equation can be obtained:

$$
E(\eta) Y=\eta Y
$$

Beam natural vibration analysis is converted to eigenvalue and eigenvector analysis of matrix $E(\eta)=M^{-1}(\eta) K(\eta)$.

Iteration method is used to calculate base frequency of beam vibration. Through circular calculations, the iteration stops when frequency relative error reaches $\kappa_{y}=\left(\omega^{i+1}-\right.$ $\left.\omega^{i}\right) / \omega^{i}<10^{-8}$.

\subsection{Result Comparison and Analysis}

6.2.1. Element Accuracy. Vibration analysis is conducted by dividing beam into one element. Table 1 shows base frequency of beam free vibration and the relative error between calculated solution and theoretical solution.

As shown in Table 1, the following results can be obtained.

(1) By applying analytical trial function element to the analysis, a more accurate solution can be attained when the relative error is less than $10^{-8}$. The series of relative error between this solution and theoretical solution is about $1 \times$ $10^{-9}$. It can be supposed that this error is calculation error of iteration efficiency.

(2) By applying interpolation trial function element to the analysis, the maximum relative error between the solution and theoretical solution reaches $30 \%$. It indicates that dynamic beam element constructed by taking polynomial function as displacement trial function has bigger error.

(3) The solution of dynamic stiffness matrix method [11] is close to theoretical solution. The relative error is almost identical with that calculated by analytical trial function method. The reason is that their displacement curves for vibrating beam are both derived from dynamic equilibrium governing equation. Element load value and node balance condition need to be analyzed in dynamic stiffness matrix method. Dynamic stiffness matrix of beam element can be directly obtained in analytical trial function method. The latter is more simple and intuitive and widely applied.

6.2.2. Element Efficiency. The beam is divided into 1 element, 2 elements, 10 elements, and 20 elements, respectively. Beam vibration numerical simulation is conducted by applying interpolation trial function method. The relative error of calculated base frequency of free vibration for every typical beam and theoretical solution is obtained. Table 2 presents the results.

As shown in Table 2, the result of interpolation trial function is closer to theoretical solution with the increasing 
of element number. But it is obvious that base frequency becomes smaller and its difference with theoretical solution is larger. The reason is that the more the elements are, the lower the structure calculation stiffness is. The greater the difference between the actual stiffness and calculation stiffness. Then the calculated base frequency further has large difference with theoretical solution. It indicates that actual deformation curve for vibrating beam is not polynomial, and polynomial function cannot be taken as displacement trial function for vibrating beam.

\section{Conclusions}

Based on the results of this investigation, the following conclusions can be drawn.

(1) The result of interpolation trial function element for simulating beam vibration is not in accordance with theoretical solution, and the relative error is larger. With element number increasing, base frequency becomes smaller and has large difference with theoretical solution. It indicates that vibrating beam displacement mode is different from polynomial mode, and the precision requirement cannot be met by taking polynomial function as displacement trial function for vibrating beam.

(2) The solution of dynamic stiffness matrix method for simulating beam vibration is close to theoretical solution. But, in this method, element load value and node balance condition need to be specially analyzed, and it is not easy to derive nonstructural question. Its application in engineering is limited.

(3) Dynamic stiffness matrix of beam element is obtained by applying analytical trial function put forward in this paper. Base frequencies of four typical beams are attained by analyzing eigenvalue and eigenvector and are compared with theoretical solution. The results show that the series of relative error is about $1 \times 10^{-9}$, and it is actually calculation error of iteration efficiency. The dynamic beam element in the light of analytical trial function put forward in this paper is highprecision element.

\section{Conflict of Interests}

The authors declare that there is no conflict of interests regarding the publication of this paper.

\section{Acknowledgments}

This research is supported by National Natural Science Funds no. 51279206, Beijing Natural Science Foundation no. 3144029, Chinese Universities Scientific Fund no. 2011JS126, and the Specialized Research Fund for the Doctoral Program of Higher Education of China no. 20110008120017.

\section{References}

[1] A. K. Clough and J. Penzien, Dynamic of Structures, McGrawHill, New York, NY, USA, 1995.
[2] Q. Jin, "An analytical solution of dynamic response for the rigid perfectly plastic Timoshenko beam," Chinese Journal of Theoretical and Applied Mechanics, vol. 16, no. 5, pp. 504-511, 1984.

[3] X. Guo, H. Chen, and Q. Zeng, "Dynamic characteristics analysis model of prestressed concrete T-type beam," Chinese Journal of Computational Mechanics, vol. 17, no. 2, pp. 176-183, 2000.

[4] D.-P. Fang and Q.-F. Wang, "Dynamic behavior analysis of an externally prestressed beam with energy method," Journal of Vibration and Shock, vol. 31, no. 1, pp. 177-181, 2012.

[5] M. Lou and T. Hong, "Analytical approach for dynamic characteristics of prestressed beam with external tendons," Journal of Tongji University: Natural Science, vol. 34, no. 10, pp. 1284-1288, 2006.

[6] J. A. Carrer, S. A. Fleischfresser, L. F. Garcia, and W. J. Mansur, "Dynamic analysis of Timoshenko beams by the boundary element method," Engineering Analysis with Boundary Elements, vol. 37, no. 12, pp. 1602-1616, 2013.

[7] J.-J. Wu, "Use of the elastic-and-rigid-combined beam element for dynamic analysis of a two-dimensional frame with arbitrarily distributed rigid beam segments," Applied Mathematical Modelling: Simulation and Computation for Engineering and Environmental Systems, vol. 35, no. 3, pp. 1240-1251, 2011.

[8] M. A. De Rosa, C. Franciosi, and M. J. Maurizi, "On the dynamic behaviour of slender beams with elastic ends carrying a concentrated mass," Computers and Structures, vol. 58, no. 6, pp. 1145-1159, 1996.

[9] R. W. Clough, "The finite element method in plane stress analysis," in Proceedings of the 2nd ASCE Conference on Electronic Computation, Pittsburgh, Pa, USA, 1960.

[10] V. Koloušek, "Anwendung des Gesetzes der virtuellen Ver schiebungen and des in der Reziprozitatssatzes," Stab weks Dynamik Lngenieur Archiv, vol. 12, pp. 363-370, 1941.

[11] Y.-Q. Long and S.-H. Bao, Structural Mechanics II, Higher Education Press, Beijing, China, 2nd edition, 1996.

[12] S. M. Hashemi and M. J. Richard, "Free vibrational analysis of axially loaded bending-torsion coupled beams: a dynamic finite element," Computers \& Structures, vol. 77, no. 6, pp. 711-724, 2000.

[13] S. Chen, M. Géradin, and E. Lamine, "An improved dynamic stiffness method and modal analysis for beam-like structures," Computers \& Structures, vol. 60, no. 5, pp. 725-731, 1996.

[14] J. R. Banerjee, H. Su, and C. Jayatunga, "A dynamic stiffness element for free vibration analysis of composite beams and its application to aircraft wings," Computers \& Structures, vol. 86, no. 6, pp. 573-579, 2008.

[15] J. R. Banerjee, S. Guo, and W. P. Howson, "Exact dynamic stiffness matrix of a bending-torsion coupled beam including warping," Computers and Structures, vol. 59, no. 4, pp. 613-621, 1996.

[16] M. Shavezipur and S. M. Hashemi, "Free vibration of triply coupled centrifugally stiffened nonuniform beams, using a refined dynamic finite element method," Aerospace Science and Technology, vol. 13, no. 1, pp. 59-70, 2009.

[17] A. K. Chopra, Dynamics of Structures, Prentice Hall, Englewood Cliffs, NJ, USA, 2000.

[18] X.-L. Hu and Z.-M. Dai, "On the dynamic stress concentrations in orthotropic plates with an arbitrary cutout," Chinese Journal of Applied Mechanics, vol. 15, no. 1, pp. 12-17, 1998. 
[19] X. Wang, L. Yang, and W. Gao, "Dynamic FEM analysis for the integration ballast structure based on variation principle," Journal of Vibration and Shock, vol. 24, no. 4, pp. 99-102, 2005.

[20] S. M. Hashemi and M. J. Richard, "A dynamic finite element (DFE) method for free vibrations of bending-torsion coupled beams," Aerospace Science and Technology, vol. 4, no. 1, pp. 4155, 2000.

[21] A. Pagani, E. Carrera, M. Boscolo, and J. R. Banerjee, "Refined dynamic stiffness elements applied to free vibration analysis of generally laminated composite beams with arbitrary boundary conditions," Composite Structures, vol. 110, no. 1, pp. 305-316, 2014.

[22] S. M. Nabi and N. Ganesan, "A generalized element for the free vibration analysis of composite beams," Computers and Structures, vol. 51, no. 5, pp. 607-610, 1994.

[23] L. Zhao and Q. Chen, "Dynamic analysis of the stochastic variational principle and stochastic finite element method for structures with random parameters," Chinese Journal of Computational Mechanics, vol. 15, no. 3, pp. 263-274, 1998.

[24] G.-H. Wang, Y.-N. Gan, and Z.-B. Wang, "Energy-variational method for the dynamic response of thin-walled I-beams with wide flange," Engineering Mechanics, vol. 27, no. 8, pp. 15-20, 2010 . 


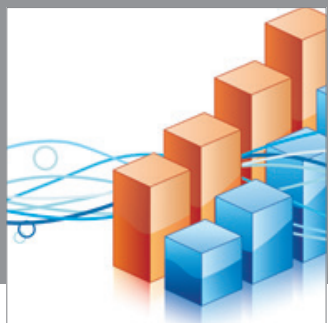

Advances in

Operations Research

mansans

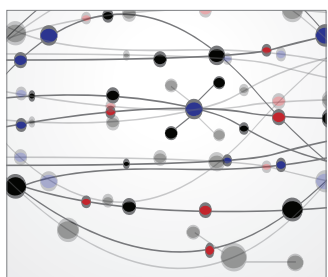

The Scientific World Journal
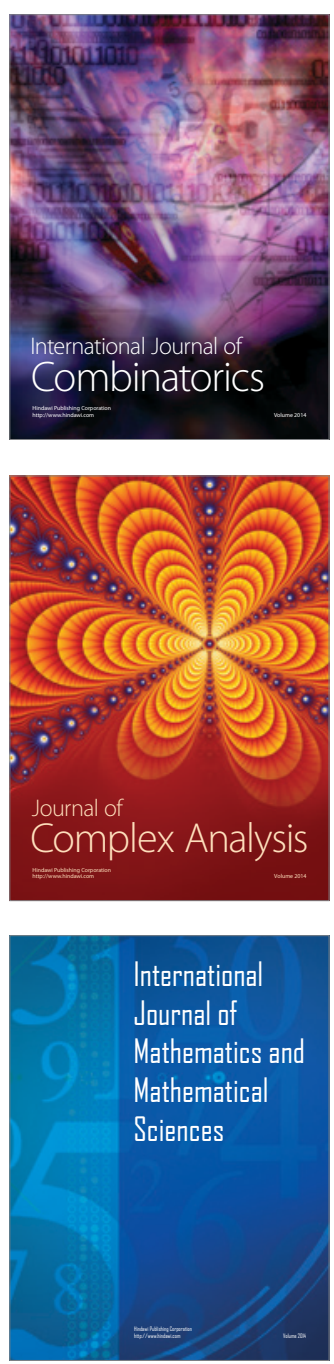
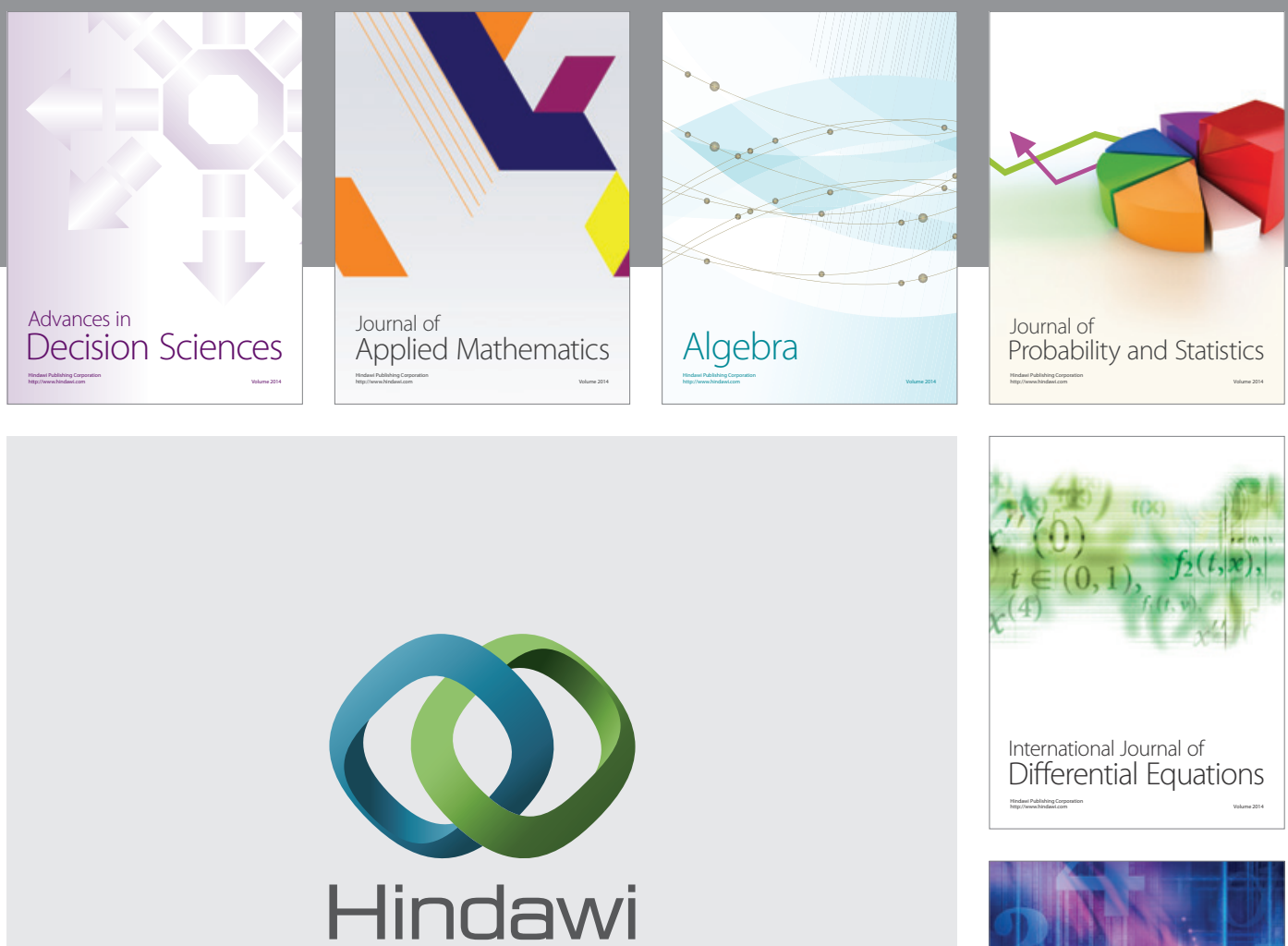

Submit your manuscripts at http://www.hindawi.com
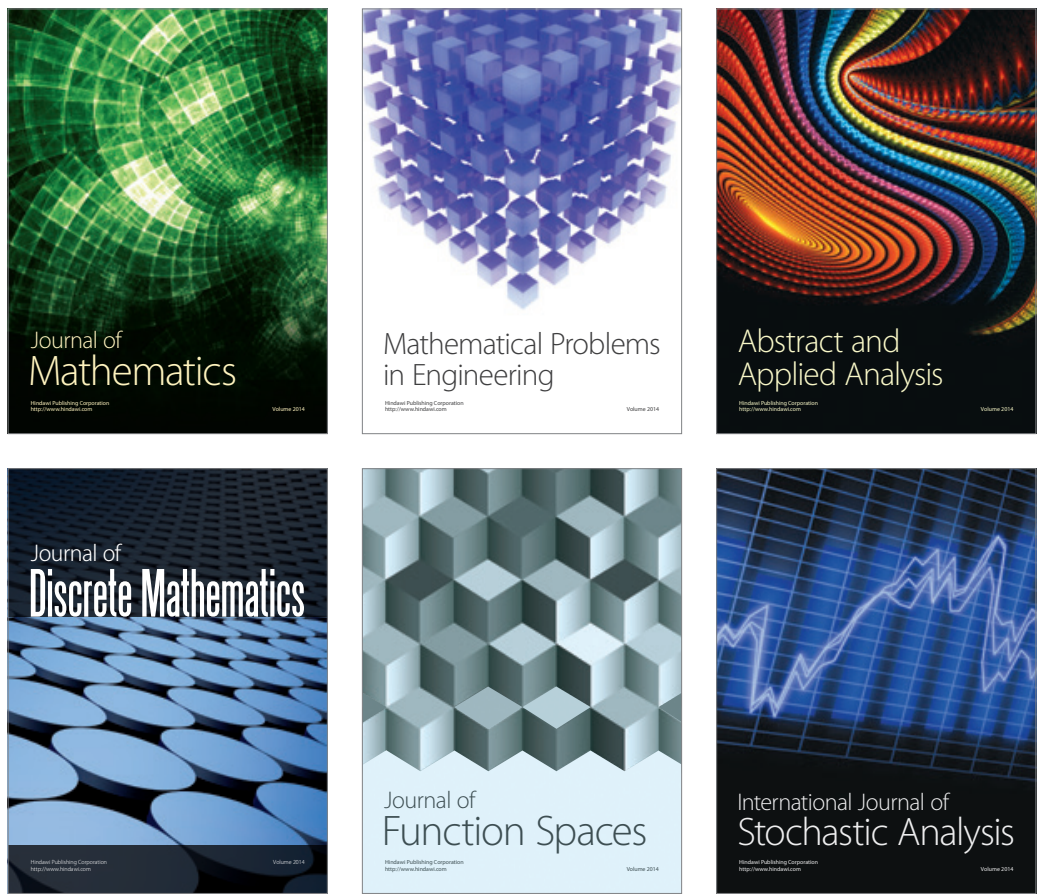

Journal of

Function Spaces

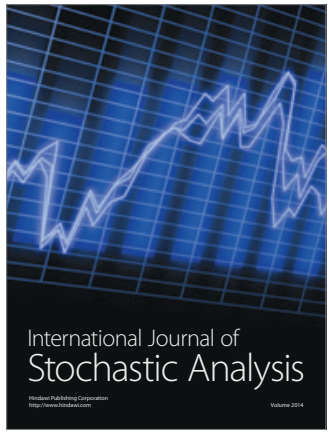

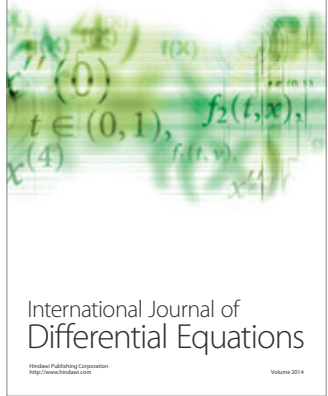
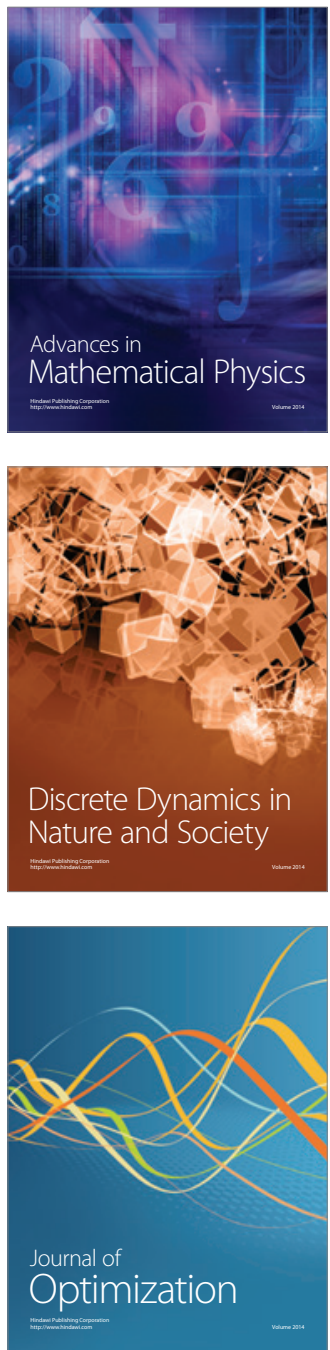